\title{
Grain boundary complexions in minerals
}

\author{
DR. PIERRE HIREL ${ }^{1}$, PHILIPPE CARREZ ${ }^{1}$ AND PROF. \\ PATRICK CORDIER ${ }^{2,3}$
}

${ }^{1}$ Univ. Lille, CNRS, INRAE, Centrale Lille, UMR 8207 -

UMET - Unité Matériaux et Transformations, F-59000 Lille, France

${ }^{2}$ Institut Universitaire de France

${ }^{3}$ Univ. Lille

Presenting Author: pierre.hirel@univ-lille.fr

Oxides are most often in polycrystalline form, hence grain boundaries are bound to play a key role in their deformation. Compared to mono-atomic metallic systems, the structure of grain boundaries in oxides is influenced by electrostatic interactions between ions. In magnesium oxide $(\mathrm{MgO})$, grain boundaries have been modeled at the atomic scale since the 1980s. Such simulations systematically predict wide open structural units as the most stable. However, these predictions are now contradicted by high-resolution electron microscopy observations, where grain boundaries always appear very compact. These observations question directly the accuracy of simulations and their ability to predict the correct structures and properties of defects.

We present the results of atomic-scale simulations on grain boundaries in $\mathrm{MgO}$. For each grain boundary studied, several metastable configurations are found, some with wide-open structural units and others with more compact structures. While the most compact structures have indeed a higher energy, we demonstrate that the absorption of vacancies stabilize them and destabilizes the wide-open structures. These results show that simulations must account for the presence of other defects in order to obtain more relevant and realistic atomic configurations. 